\title{
Modular Model Predictive Control Concept for Building Energy Supply Systems: Simulation Results for a Large Office Building
}

\author{
Barbara Mayer ${ }^{1}$ Michaela Killian ${ }^{2}$ Martin Kozek ${ }^{2}$ \\ ${ }^{1}$ Institute of Industrial Management,FH Joanneum, Austria, \{barbara mayer $\} @ f h-j o a n n e u m$. at \\ ${ }^{2}$ Institute of Mechanics and Mechatronics, Vienna University of Technology, Austria, \{michaela killian, \\ martin kozek\} @tuwien.ac.at
}

\begin{abstract}
The management of modern large buildings' energy supply systems (ESS) demands the maximal usage of renewable energy sources at minimum energy costs while meeting the energy demand of the consumption zone. Building ESS for heating and cooling usually consist of various supply circuits with several energy sources and different physical characteristics, possibly incorporating switching aggregates (heat pump, chiller) with latency times and stratified storage which change their operating state in a discontinuous fashion. Hence, these circuits can be seen as hybrid systems whose modelling as well as optimisation are demanding. Model predictive controllers (MPC) are an effective means for the optimisation of such problem formulations with divergent goals. The proposed modular predictive control concept (MPCC) is designed for a flexible usage in ESS building automation adding one appropriate MPC for each supply circuit including mixed-integer MPCs to the individual building's control structure. The resulting MPCC is capable of maximising the usage of renewable energy sources at minimum cost as well as efficiently managing switching aggregates with active storage. Suitable modelling of the linear and hybrid systems is demonstrated and validated on the example of a large office building in Austria. Furthermore, a simulation study shows the performance of the resulting MPC concept compared to a rule-based controller.
\end{abstract}

Keywords: building energy supply system, model predictive control

\section{Introduction}

Building control has become an important field since the building sector is responsible for about $40 \%$ of the total energy consumption in developed countries, (Pérez-Lombard et al., 2008). Therefore, an increasing effort has been put both on the development of energy saving building physical structure such as passive heating and cooling systems and on energy efficient building operation techniques. Model predictive control (MPC) has been proven as a promising technology for such building systems in recent years, (Široky et al., 2011), with the ability of incorporating most influential quantities such as weather forecasts, (Oldewurtel et al., 2012), or occupancy information as well as technical constraints into the prediction. Moreover, the controller's optimisation target can include conflicting objectives expressed in thermal comfort and economic trade off. However, modelling of building systems is a crucial part for predictive building control, (Privara et al., 2013).

The efficient operation of large buildings' energy supply systems (ESS) aims at a maximal usage of renewable energy sources at minimum energy costs. Energy supply systems (ESS) of large buildings usually consist of various supply circuits of different physical characteristics, including heat exchangers and continuous pumps or additionally switching aggregates such as chillers or heat pumps with successive active energy storage, e.g. stratified storage tanks. Obtaining accurate models of latter systems is difficult, (Liu and Henze, 2004), since stratified storage is operated in either charging or discharging mode which is directly influenced by the state of the aggregate and its limitation of minimal up and down times. Due to the mixture of continuous and discrete variables hybrid models are suitable to approximate those systems. The efficient control of hybrid systems is challenging. For the optimisation within a predicitve controller with quadratic target this leads to a constrained mixed-integer quadratic problem (MIQP) at each time step. This can either be solved by a dual stage procedure where the storage tank operation mode profile is firstly fixed and the remaining QP solved in a second step, (Ma et al., 2009), or in a single step e.g. by using a branch and bound algorithm, (Mayer et al., 2016).

The classic approach to controlling buildings, especially ESS, is rule based control (RBC) due to its simplicity. However, RBC does not allow advanced management of e.g. active storage. Advanced control approaches of the recent years propose one single MPC controlling the entire building comprising the buildings' zone control as well as the energy supply optimisation, (Oldewurtel et al., 2012; Privara et al., 2013). While the operation with one global MPC would guarantee optimality, it is too rigid for application in industrial building automation.

This work introduces a modular predictive control concept (MPCC) for the energy supply level using dedicated predictive controllers for the respective circuits' physical characteristics. Basic circuits with heat 
exchangers and continuous pumps can be approximated by linear state space systems, whereas the hybrid models are represented by piecewise affine (PWA) systems. All models are analytically derived based on thermodynamic principles and energy balance equations, (Berkenkamp and Gwerder, 2014; Mayer et al., 2015). The overall structure is capable of optimising heating and cooling supply at minimum cost and maximal usage of renewable energy sources. Furthermore, an efficient management of the active storage connected with the switching aggregate is guaranteed due to the application of a dedicated mixed-integer MPC (MI-MPC). The MPCC is designed to be embedded into a hierarchical building control structure, decoupling the energy consuming office level (OL) from the energy supply system level. In the OL a predictive controller is assumed to maximise user comfort while minimising the supply energy respecting disturbances, e.g. (Killian et al., 2015). It thus provides the reference trajectory for heating and cooling power for the MPCC. The modularity of the MPCC enables a flexible application in industrial building automation using dedicated models and MPCs allowing separate implementation and tuning. The main contributions of the paper therefore are:

- A modular predictive control concept (MPCC) for building energy supply systems (ESS) maximising the usage of renewable energy sources at minimum cost.

- Supply circuits with switching aggregates and stratified storage are modelled as hybrid systems and controlled by MI-MPCs aiming at an efficient management of aggregates and storage.

- Flexible application in industrial building automation leading to an application specific control architecture.

- A simulation study showing the performance of the proposed MPCC compared to a rule-based controller applied to a demonstration building in Austria.

The remainder of this paper is structured as follows: In Section 2 the demonstration building setup as well as the overall control structure is given. The building models for the ESS dedicated for the different MPCs are explained in Section 3, whereas the predictive controllers are introduced in Section 4. The simulation results for the demonstration building are shown in Section 5 and finally, a conclusion is drawn in Section 6.

\section{Modular Model Predictive Control Concept}

Within this Section the building setup and the overall MPCC for the demonstration building is given.

\subsection{Building Setup}

The building which is presented in this work is a $27.000 \mathrm{~m}^{2}$ University building in the center of Salzburg, Austria. It has five floors above ground containing several large and numerous smaller meeting rooms, offices and lecture rooms. For this work focus is put on the second and third floor, which comprise of about 500 rooms of some $13.000 \mathrm{~m}^{2}$, almost all used as offices. Both floors are supplied by Fan Coils (FC) and a Thermal Activated Buildings System (TABS). The ESS of this building consists of heating and cooling supply circuits for FC and TABS, see Figure 1. The FC supply is split into cooling supply based

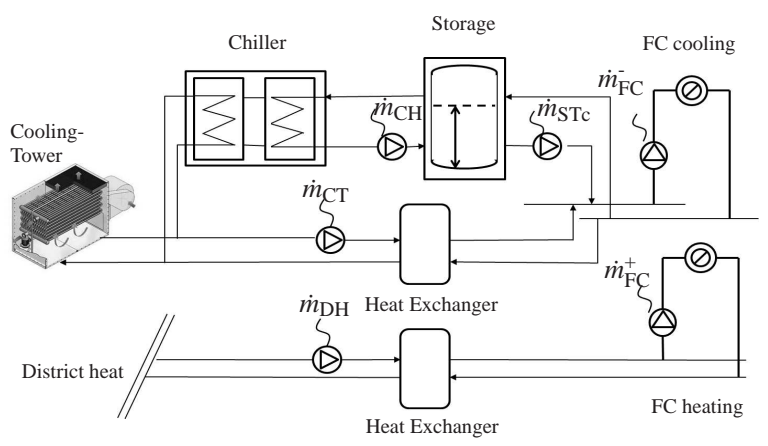

(a) FC circuits including free cooling, the chiller, and district heat.

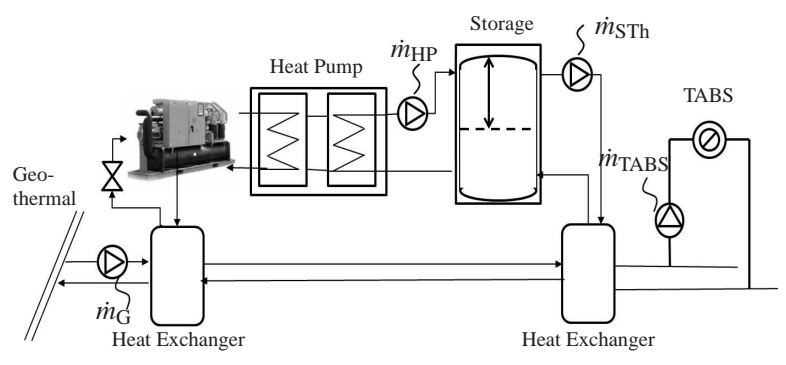

(b) TABS circuits including geothermal source and the heat pump.

Figure 1. Energy supply circuits for cooling and heating of the University building in Salzburg, Austria.

on free cooling and chiller circuit and heating supply from the district heat. TABS has only one piping system supplied by the geothermal source and is routed via the heat pump circuit in case of heating.

\subsection{Modular Predictive Control Concept}

The modular predictive control concept (MPCC) generally consists of independently acting MPCs with the same target - one MPC for each supply circuit comprising the corresponding energy source as well as the supply system of the building. Basic circuits consisting of heat exchangers and continuous pumps can be approximated by linear models. Consequently, linear MPCs (LMPC) are applicable in this case. However, if switching aggregates have to be considered, the predicitve controller not only has to optimise the continuous manipulated variables but also the discrete aggregate's switching state. Moreover, the coupled active storage changes its operating mode in 
a discontinuous fashion depending on the switching instance. Therefore, a dedicated mixed-integer MPC (MIMPC) is applied optimising latter circuits considered as hybrid systems. The control structure for the proposed ESS, see Figure 2, is designed according to the available energy sources and aggregates shown in Figure 1(a) and Figure 1(b) which distinguishes between FC and TAB$\mathrm{S}$ heating and cooling supply. For the FC cooling sys-

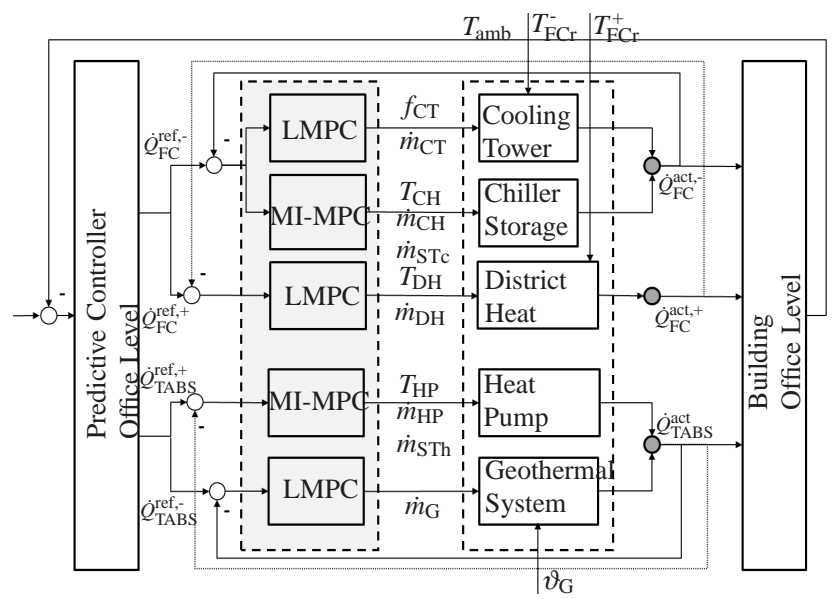

Figure 2. Control structure for cooling and heating circuits for FC and TABS embedded into a hierarchical building control structure.

tem an LMPC controls the free cooling circuit, whereas an MI-MPC manages the cooling supply from the chiller including a stratified storage tank. The FC heating supply is sourced by the district heat and controlled by the second LMPC. The TABS is supplied by the geothermal source either used directly in the case of cooling or routed via the heat pump and subsequent stratified storage tank if heating is required. Hence, an LMPC is active for cooling and an MI-MPC for heating. Furthermore, the ESS control structure is embedded into an overall hierarchic building control structure with a predicitve controller for the OL, e.g. (Killian et al., 2015), which is responsible for providing a prediction of the required heating and cooling power, $\dot{Q}_{\mathrm{FC}}^{\text {ref,+ }}$ respectively $\dot{Q}_{\mathrm{FC}}^{\text {ref,- }}$, for each energy supply system. All variables and parameters used are listed in Table 1 and the two types of MPCs are formally described in Section 4.

\section{Energy Supply Level - ESS - Models}

In this Section the modelling strategy and structure for the circuits of the ESS shown in Figure 1 is given. Each circuit is modelled individually.

\subsection{Linear Models}

For building control aspects the achievable heating or cooling power, $\dot{Q}$, of each supply circuit is relevant, which can be approximated by simplified energy balance equations: $\dot{Q}=\dot{m} \cdot \Delta T \cdot c p$, where $\dot{m}$ denotes the mass flow, $\Delta T$ the difference of supply and return water temperature, and $c p$ the specific heat capacity of water. Heat ex-
Table 1. Nomenclature.

\begin{tabular}{lll}
\hline Variable & Description & Unit \\
\hline$\dot{m}_{\mathrm{CT}}$ & mass flow from cooling tower & {$[\mathrm{kg} / \mathrm{s}]$} \\
$f_{\mathrm{CT}}$ & fan speed of cooling tower & {$[\mathrm{m} / \mathrm{s}]$} \\
$T_{\mathrm{amb}}$ & ambient temperature & {$\left[{ }^{\circ} \mathrm{C}\right]$} \\
$T_{\mathrm{FCr}}^{-}$ & return temperature for FC cooling & {$\left[{ }^{\circ} \mathrm{C}\right]$} \\
$T_{\mathrm{CH}}$ & supply temperature of chiller & {$\left[{ }^{\circ} \mathrm{C}\right]$} \\
$\dot{m}_{\mathrm{CH}}$ & mass flow from chiller to storage & {$[\mathrm{kg} / \mathrm{s}]$} \\
$\dot{m}_{\mathrm{STc}}$ & mass flow from storage of cooling circuit & {$[\mathrm{kg} / \mathrm{s}]$} \\
$T_{\mathrm{DH}}$ & supply temperature of district heat & {$\left[{ }^{\circ} \mathrm{C}\right]$} \\
$\dot{m}_{\mathrm{DH}}$ & mass flow from district heat & {$[\mathrm{kg} / \mathrm{s}]$} \\
$T_{\mathrm{FCr}}^{+}$ & return temperature for FC heating & {$\left[{ }^{\circ} \mathrm{C}\right]$} \\
$T_{\mathrm{HP}}$ & supply temperature of heat pump & {$\left[{ }^{\circ} \mathrm{C}\right]$} \\
$\dot{m}_{\mathrm{HP}}$ & mass flow from heat pump to storage & {$[\mathrm{kg} / \mathrm{s}]$} \\
$\dot{m}_{\mathrm{STh}}$ & mass flow from storage of heating circuit & {$[\mathrm{kg} / \mathrm{s}]$} \\
$\dot{m}_{\mathrm{G}}$ & mass flow from geothermal source & {$[\mathrm{kg} / \mathrm{s}]$} \\
$\vartheta_{\mathrm{G}}$ & difference geothermal supply, return temp. & {$\left[{ }^{\circ} \mathrm{C}\right]$} \\
$\dot{Q}_{j}^{\text {ref,+ }}$ & reference heat flow for supply $j$ heating & {$[\mathrm{kW}]$} \\
$\dot{Q}_{j}^{\text {ref,- }}$ & reference heat flow for supply $j$ cooling & {$[\mathrm{kW}]$} \\
$\dot{Q}_{j}^{\text {act, }+}$ & actual heat flow for supply $j$ heating & {$[\mathrm{kW}]$} \\
$\dot{Q}_{j}^{\text {act,- }}$ & actual heat flow for supply $j$ cooling & {$[\mathrm{kW}]$} \\
$j$ & supply: TABS or FC & \\
\hline
\end{tabular}

changers and continuous pumps preferably work around a certain operating point. For the geothermal and the district heat supply two linearised static models based on those thermodynamic principles are analytically derived. The geothermal model contains one manipulated variable, $\dot{m}_{\mathrm{G}}$, one disturbance, $\vartheta_{\mathrm{G}}$, and the output $\dot{Q}_{\mathrm{TABS}}^{-}$. The $\Delta$ variables denote the deviation of the variables to the operating point and $\mathrm{COP}_{\mathrm{G}}$ the coefficient of the geothermal performance:

$$
\Delta \dot{Q}_{\mathrm{TABS}}^{-}=\underbrace{\mathrm{COP}_{\mathrm{G}} \cdot \mathrm{cp}}_{\text {const. }} \cdot\left[\left.\dot{m}_{\mathrm{G}}\right|_{o} \cdot \Delta \vartheta_{\mathrm{G}}+\left.\vartheta_{\mathrm{G}}\right|_{o} \cdot \Delta \dot{m}_{\mathrm{G}}\right] .
$$

The district heat model is derived in the same way and contains two manipulated variables, $T_{\mathrm{DH}}$ and $\dot{m}_{\mathrm{DH}}$, one disturbance, $T_{\mathrm{FCr}}^{+}$, and the output $\dot{Q}_{\mathrm{FC}}^{+}$:

$$
\begin{aligned}
& \Delta \dot{Q}_{\mathrm{FC}}^{+}=\underbrace{\mathrm{COP}_{\mathrm{DH}} \cdot \mathrm{cp}}_{\text {const. }} \cdot\left[\left.\dot{m}_{\mathrm{DH}}\right|_{o} \cdot\left(\Delta T_{\mathrm{DH}}-\Delta T_{\mathrm{FCr}}^{+}\right)\right. \\
& \left.+\left(\left.T_{\mathrm{DH}}\right|_{o}-\left.T_{\mathrm{FCr}}^{+}\right|_{o}\right) \cdot \Delta \dot{m}_{\mathrm{DH}}\right] .
\end{aligned}
$$

As depicted in Figure 1(a) free cooling is based on the cooling tower. Free cooling is exclusively used if the chiller is inactive. The ambient temperature, $T_{\mathrm{amb}}$, constrains the cooling tower's operation for free cooling which is also a main disturbance next to the return water temperature $T_{\mathrm{FCr}}^{-}$. Modelling cooling towers analytically aims at detailed complex models with non-linear dynamics of high order which are not suitable for the usage within MPCs. Hence, black-box identification is expedient if 
historic data of the cooling tower in operation is available. Since the cooling tower can be operated at two fan speeds $i \in\{1,2\}$, the system can be approximated by two linear static models:

$$
\dot{Q}_{\mathrm{FC}}^{-}(i)=c_{i, 1} \cdot T_{\mathrm{amb}}+c_{i, 2} \cdot T_{\mathrm{FCr}}^{-}+c_{i, 3} \cdot \dot{m}_{\mathrm{CT}}+c_{i, 4},
$$

where the corresponding coefficients $c_{i, k}$ have to be estimated within the black-box identification routine using historic data of the free cooling system. Note that the model in Eq. (3) is linear in the parameters $c_{i, j}$, thus least squares methods can be employed for optimal parameter estimation, (Ljung, 1999).

\subsection{Hybrid Models}

The chiller and the heat pump are switching aggregates with latency times, such that minimum up and down times have to be respected, e.g. after switching on the aggregate it must operate for a minimum up time until it can be shut off again and vice versa. Furthermore, the stratified storage tanks can operate in two basic modes: charging and discharging. Each mode is represented by a dedicated model with continuous variables. The discrete switching state of the aggregate (on/off), $l_{\text {on }}$, specifies which mode is exclusively active at each time. These operation modes further depend on the difference of the mass flows to, $\dot{m}_{l}$, and from, $\dot{m}_{\mathrm{STk}}$, the storage tank where $l=\{\mathrm{CH}, \mathrm{HP}\}$ denotes the aggregate and $k=\{\mathrm{c}, \mathrm{h}\}$ the cooling or heating circuit, (Mayer et al., 2015). The hybrid dynamic models contain three continuous states, $x(t)$, namely the thermocline of the storage $z_{k}(t)$, the temperature of the cold respective hot water $T_{k}(t)$, and the cooling respective heating power $\dot{Q}_{j}(t)$. The three continuous manipulated variables, $u(t)$, are the supply temperature of the aggregate $T_{l}(t)$, the mass flow from the aggregate to the storage $\dot{m}_{l}(t)$, and the mass flow from the storage to the TABS or FC supply $\dot{m}_{\mathrm{STk}}(t) . l_{\mathrm{on}}(t)$ is the discrete manipulated variable representing the switching state of the corresponding aggregate $l$ for each time $t$. The continuous output $y(t)=\dot{Q}_{j}(t)$ denotes the cooling respective heating power for the supply $j$ to the building. For each operating mode a model is firstly analytically derived through first order differential equations and secondly linearised at a fixed operating point. The non-linear dynamics can then be approximated by a hybrid system state-space formulation with discrete as well as continuous inputs represented by a piecewise affine (PWA) system:

$$
\begin{aligned}
& x(t+1)=A_{h} x(t)+B_{h} u(t), \quad \text { if } \delta_{h}(t)=1, \\
& y(t+1)=C x(t),
\end{aligned}
$$

where the binary variables $\delta_{h}(t) \in\{0,1\}, \forall h=1, . ., 3$, are introduced to denote the status of the operation mode $h$ and the system matrices are $A_{h} \in \mathbb{R}^{3 \times 3}$ and $B_{h} \in \mathbb{R}^{3 \times 4}$. $C=\left[\begin{array}{lll}0 & 0 & 1\end{array}\right]$ for all three modes since the third state is equal to the output. The explicit matrices for the chiller circuit model are given in (Mayer et al., 2016), whereas for the modelling of the heat pump circuit the reader is referred to (Mayer et al., 2015).

\section{Model Predictive Controllers}

The formal description of the two MPC types used within the MPCC and their common objective are given in this Section.

\subsection{Objective Function}

The predictive controller for the OL, e.g. (Killian et al., 2015), is responsible to guarantee user comfort by keeping the indoor temperature within a certain tolerance at minimum energy demand. The objective for the ESS on the other hand is to minimise the deviation to the required power and the energy costs.

For all MPC implementations a quadratic optimisation target is used, where the deviation to the reference trajectory of the cooling respective heating power is penalised with factor $Q_{y}$. Furthermore, the energy costs represented by $Q_{u}$ caused by the manipulated variables are taken into account. Both additive terms are considered for each time step $t$ over the whole prediction horizon $N p$. The $\Delta$ variables again denote the deviation from the corresponding operating point. The formal description of the objective is given by:

$$
J^{\star}=\min _{\Delta u \in U} \sum_{t=0}^{N p-1}\left\|\Delta \dot{Q}_{j}^{\mathrm{ref}}-\Delta \dot{Q}_{j}^{\mathrm{act}}\right\|_{Q_{y}}^{2}+\|\Delta u(t)\|_{Q_{u}}^{2},
$$

\subsection{LMPC}

The three LMPCs for this work have a quadratic optimisation target as presented in Eq. (5). They only differ in the model they rely on. The FC model for free cooling uses a linear model with absolute inputs, whereas the TAB$\mathrm{S}$ geothermal model and the FC district heat model are a linearised model with $\Delta$ - variables which denote the deviation from the corresponding operating point. All three LMPCs are implemented according to the receding horizon strategy, (Camacho and Alba, 2013).

\subsection{MI-MPC}

In Section 3 the hybrid models for the chiller and the heat pump circuits are motivated. The objective is given in Section 4.1. The corresponding constraints are:

PWA model as given in Eq. (4),

$$
\begin{aligned}
& \delta_{h}(t) \in\{0,1\} \\
& \sum_{h} \delta_{h}(t)=1 \\
& u_{\min } \leq u(t) \leq u_{\max } \\
& x_{\min } \leq x(t) \leq x_{\max } \\
& l_{\mathrm{on}}(t)-l_{\mathrm{on}}(t-1) \leq l_{\mathrm{on}}\left(\omega_{\mathrm{up}}\right),(\text { Off/On switch }) \\
& l_{\mathrm{on}}(t-1)-l_{\mathrm{on}}(t) \leq 1-l_{\mathrm{on}}\left(\omega_{\text {down }}\right),(\text { On/Off switch })
\end{aligned}
$$

$\forall j \in\{\mathrm{TABS}, \mathrm{FC}\}$ and $l \in\{\mathrm{CH}, \mathrm{HP}\}$, where $u_{\min }$ and $u_{\max }$, respectively $x_{\min }$ and $x_{\max }$, denote the the capacity limits of the manipulated variables and the physical bounds of the stratified storage. The constraints on latency times 
with minimum up and down times are given in Eq. (6e) and Eq. (6f) with $\omega_{\text {up }}=t, t+1, \ldots, \min \left(t+N p, t+T_{l}^{\text {up }}-1\right)$ if we consider the minimum up time and $\omega_{\text {down }}=t, t+$ $1, \ldots, \min \left(t+N p, t+T_{l}^{\text {down }}-1\right)$ in the other case. Eq. $(6 \mathrm{~b})$ denotes that at each time only one hybrid mode can be active. Since the objective given in Eq. (5) is quadratic the resulting optimisation problem is a mixed-integer quadratic problem (MIQP) to be solved each time step $t=0, \cdots, N p-1$. For this work a branch and bound algorithm is applied, (Mayer et al., 2016), which relaxes the original problem by replacing integrality constraints, (6a), i.e. integer and particularly binary variables are allowed to span over the whole continuous interval, aiming at solving many QPs and searching for the best solution.

\section{Simulation Results}

In this Section the simulation setup is given. Furthermore, simulation results showing the performance of the MPC$\mathrm{C}$ are discussed and the comparison results to a simuated rule-based controller (RBC) are given.

\subsection{Simulation Setup}

The simulation for the demonstration building described in Section 2.1 is based on a snapshot of historic data of the implemented automation system for the disturbances ambient temperature and $\vartheta_{\mathrm{G}}$. The simulation is shown from the beginning of September until the end of November 2014. The comparison between MPCC and RBC is given for all seasons 2014. The prediction horizon $N p$ for all predictive controllers is 12 hours and the sampling time is one hour. The return water temperature for the cooling FC circuit, $T_{\mathrm{FCr}}^{-}$, is $20^{\circ} \mathrm{C}$, whereas for the heating circuit the return water temperature, $T_{\mathrm{FCr}}^{+}$, is $24^{\circ} \mathrm{C}$. The two stratified storage tanks have a volume of almost $16 \mathrm{~m}^{3}$ each.

\subsection{Demonstration of MPCC Performance}

The simulation study shows the performance of the proposed modular MPC concept for the demonstration building. For this work, the power demand trajectories are given by the OL predictive controller introduced in (Killian et al., 2015) for both TABS and FC supply. Since there is only one piping system for TABS, the predicted power is split into a negative cooling, $\dot{Q}_{\mathrm{TABS}}^{\text {ref,- }}=$ $\min \left(\dot{Q}_{\mathrm{TABS}}^{\mathrm{ref}}, 0\right)$, and a positive heating trajectory, $\dot{Q}_{\mathrm{TABS}}^{\mathrm{ref},+}=$ $\max \left(\dot{Q}_{\mathrm{TABS}}^{\text {ref }}, 0\right)$. For the FC system the reference trajectories are already split by the OL controller. The simulation for all MPCs, the LMPCs as well as the MI-MPCs, is run simultaneously. However, for the FC cooling supply two circuits are implemented but only one can be active at a time (either chiller or free cooling). Therefore, an additional rule is applied which prefers the usage of the renewable energy source respectively of the corresponding LMPC if the ambient temperature does not exceed $18^{\circ} \mathrm{C}$.

Figure 3 shows the ambient temperature profile for the simulation period from September to November 2014. One can see a drop of the mean temperature after hour
1200 by about $7^{\circ} \mathrm{C}$. The green line depicts the technical

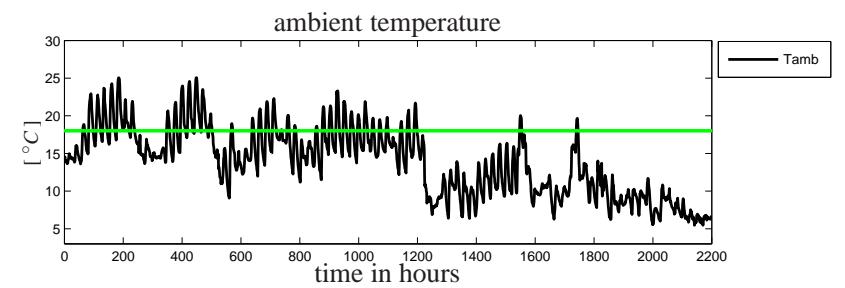

Figure 3. Ambient temperature profile starting with 1st Sept. 2014.

limit for the usage of the free cooling circuit.

Figure 4 shows the simulation results for the TABS circuits, where according to the ambient temperature profile in the first half of the simulation period cooling is requested by the OL, represented by $\dot{Q}_{\mathrm{TABS}}^{\text {ref,- }}$. The temperature spread of $\vartheta_{\mathrm{G}}$ is sufficient to provide the required cooling energy only from the geothermal source, the manipulated variable $\dot{m}_{\mathrm{G}}$ for the supply of $\dot{Q}_{\mathrm{TABS}}$ is given in the second subplot. The heat pump is inactive over large parts of this first simulation period which can be seen at the red line in the last subplot. From hour 1200 onwards heating is requested by the OL, $\dot{Q}_{\mathrm{TABS}}^{\mathrm{ref},+}$, and supplied by the heat pump circuit with perfect fit. The temperature of the water supply from the heat pump as well as the pumps of the heat pump circuit are presented in the second subplot.

Figure 5 shows the simulation results for the FC circuits. The references for cooling, $\dot{Q}_{\mathrm{FC}}^{\text {ref,- }}$, and heating, $\dot{Q}_{\mathrm{FC}}^{\text {ref,+ }}$, are computed by the OL controller. The first subplot shows the performance of the MI-MPC and LMPCs for the FC circuits. In the second and third subplot the manipulated variables are presented. The red lines denote the contributions to FC heating supply of the district heat circuit, whereas the blue and grey lines show the temperature and mass flows for the chiller circuit. The green line in subplot three depicts the mass flow of the pump from the cooling tower of the free cooling circuit. The last subplot indicates which cooling circuit is active. Note, that free cooling is always active if the technical condition $T_{\mathrm{amb}} \leq 18^{\circ} \mathrm{C}$ is fulfilled. Since the energy demand for the cooling FC circuit is low, the chiller is not regularly active, even if free cooling cannot be activated. Due to the specific hydraulic architecture of the demonstration building the pump supplying the cold water from the stratified storage tank has to be activated if free cooling is not active. Thus, a minimal deviation to $\dot{Q}_{\mathrm{FC}}^{\text {ref,- }}$ has systematically be taken into account in these periods (see blue line in first subplot in Figure 5).

\subsection{Comparison between MPCC and RBC}

ESS are conventionally controlled by rule-based controllers (RBC). The RBC of the demonstration building is also simulated for comparison: Free cooling is also preferred but it may only be active if ambient temperature has been below $18^{\circ} \mathrm{C}$ for the past three hours. The chiller has to be switched on if the low storage temperature is higher than $12^{\circ} \mathrm{C}$ and switched off if it is lower than $7^{\circ} \mathrm{C}$. 

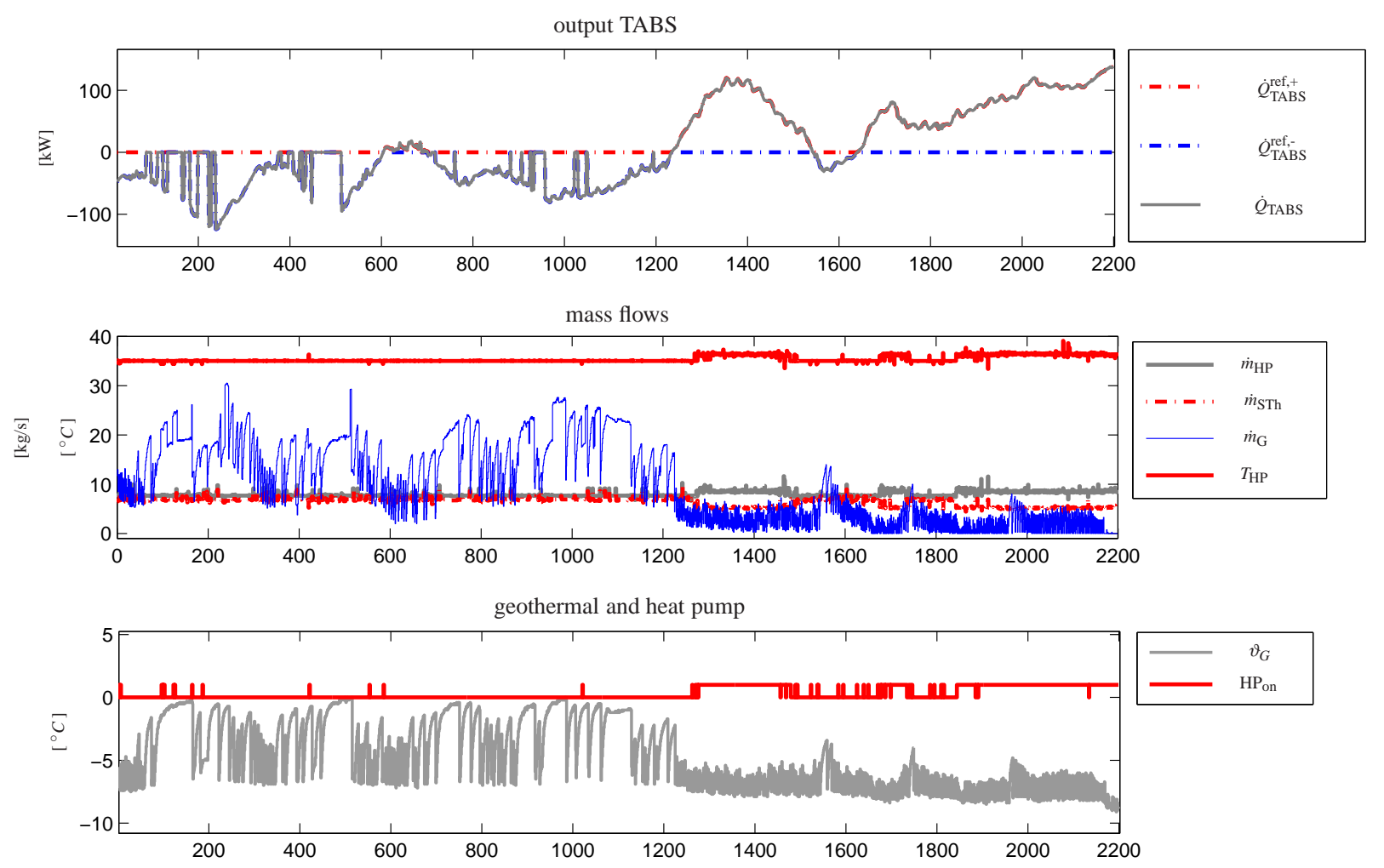

Figure 4. Simulation results for the TABS circuits starting with 1st Sept. 2014.
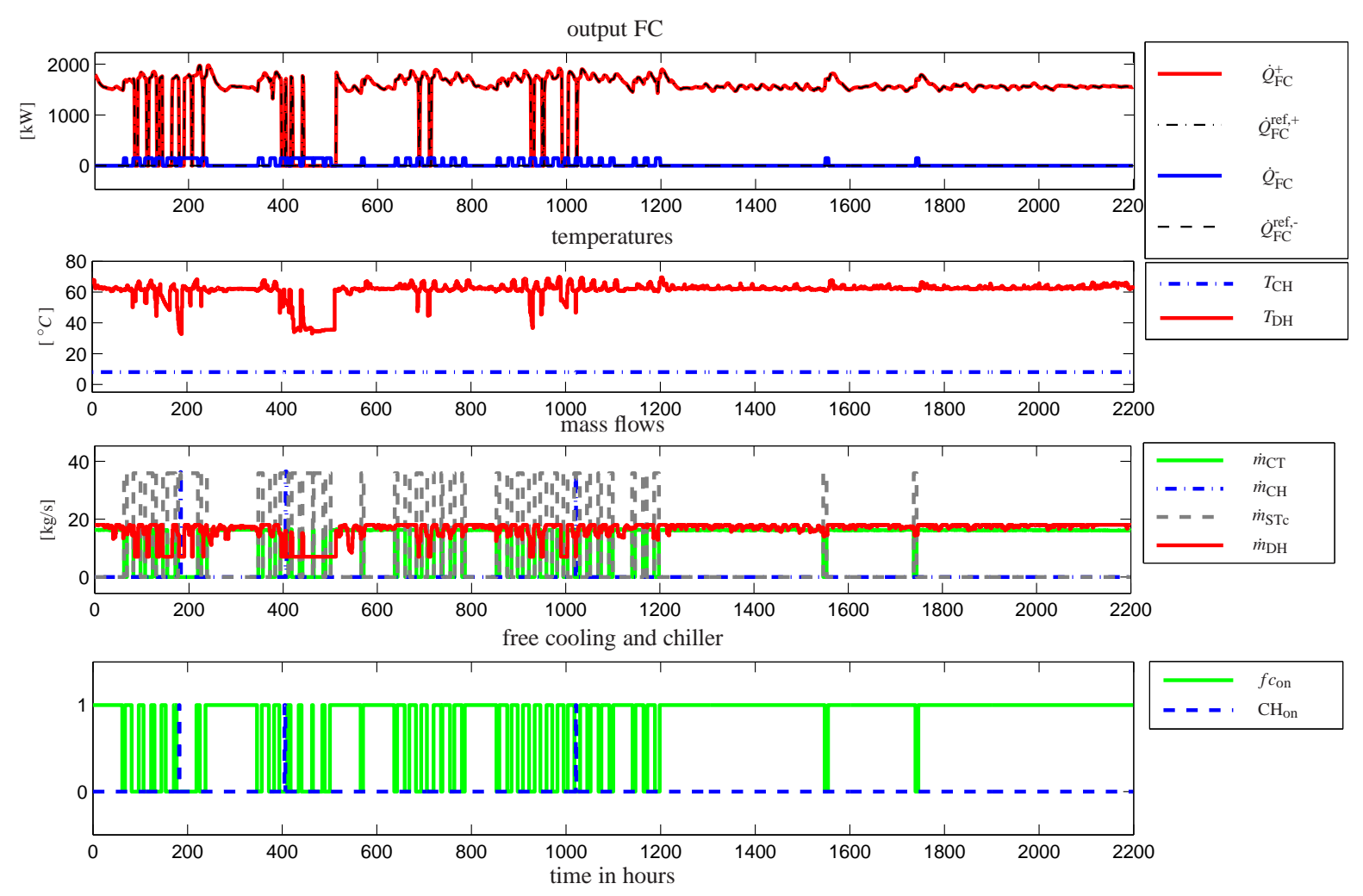

Figure 5. Simulation results for the FC circuits starting with 1st Sept. 2014. 
Compared to this RBC the proposed MPCC achieves an increase of free cooling hours of almost 5\% in winter (January-March and December 2014) up to over 30\% in summer (June-August 2014). About $30 \%$ to $40 \%$ more cooling power $\dot{Q}^{-}$is supplied via free cooling using the MPCC. The number of transitions of the chiller from state off to on can be reduced by around $15 \%$ in summer up to $60 \%$ in transition period (April-May and SeptemberNovember 2014), which is equivalent to a significant reduction for maintenance cost for the aggregate. Table 2 shows the precise figures of the MPCC in percentage of the RBC. Simulation results show the best results for the

Table 2. Performance of the MPCC compared to the RBC in percentage according to the seasons.

\begin{tabular}{l|c|c|c}
\hline season & fc hours & $\dot{Q}^{-}$via fc & chiller state trans. \\
\hline winter & 104.7 & 130.5 & 0.0 \\
transition & 108.4 & 134.9 & 39.1 \\
summer & 132.6 & 141.2 & 84.8 \\
\hline
\end{tabular}

MPCC cooling supply during summer, where the predictive character of the control concept is most effective compared to the conventional controller.

Relaxing the upper limit of $T_{\mathrm{amb}} \leq 18^{\circ} \mathrm{C}$ for the extended usage of free cooling leads to an average increase of mass flow of the cooling tower $\dot{m}_{\mathrm{CT}}$ at lower variance. Table 3 lists the simulation results for the upper limit varying from $18^{\circ} \mathrm{C}$ to $22^{\circ} \mathrm{C}$ for autumn 2014 . The number of free cooling hours is given in percentage of the simulation hours.

Table 3. Effect of a relaxation of the upper limit $T_{\text {amb }}$ on $\dot{m}_{\mathrm{CT}}$.

\begin{tabular}{l|c|c|c}
\hline upper limit & fc hours [\%] & mean $\dot{m}_{\mathrm{CT}}$ & std. dev. $\dot{m}_{\mathrm{CT}}$ \\
\hline $18^{\circ} \mathrm{C}$ & 89.1 & 21.8 & 8.2 \\
$20^{\circ} \mathrm{C}$ & 96.2 & 23.4 & 5.6 \\
$22^{\circ} \mathrm{C}$ & 99.5 & 24.0 & 3.6 \\
\hline
\end{tabular}

The proposed MPCC allows to pursue different strategies for heating and cooling control, depending on the parametrisation of the weighting factors of the optimisation targets. For this work emphasis was put on the maximal usage of renewable energy sources with minimum cost while assuring minimal deviation of the delivered cooling and heating power for the supply systems in order to maximise the user comfort in the OL. The simulation results show, that the proposed MPCC is able to meet the requirements of the OL predictive controller almost perfectly and to maximise the usage of renewable energy sources such as free cooling. Furthermore, maintenance cost can be reduced due to a reduction of state transitions of the switching aggregates.

\section{Conclusions}

This paper introduces a modular predictive control concept (MPCC) for modern energy supply systems (ESS) of large buildings with several energy sources and supply circuits. Due to its modularity the concept is flexibly applicable for industrial building's ESS control, which is shown on a demonstration building in Austria. The resulting MPCC includes linear MPCs as well as mixed-integer MPCs (MI-MPC) dedicated for the efficient control of basic circuits respectively those with switching aggregates such as chillers with active storage. The simulation study shows that the proposed MPCC is able to accurately deliver a prescribed cooling respective heating power trajectory at minimum cost and a maximal usage of renewable energy sources. The MPCC is capable to maximise the usage of free cooling and - due to the MI-MPC - to efficiently manage the switching aggregates: compared to a RBC simulation results show an increase of free cooling hours of up to $30 \%$ and a reduction of the chiller's transitions from state off to on by up to $60 \%$ aiming at a consequent decrease of maintenance cost depending on the respective season of the year. Simulation results of the MPCC are therefore very promising for the future implementation in the demonstration building.

\section{Acknowledgment}

This work was supported by the project "SMART MSR" (FFG, No. 832103) in cooperation with evon GmbH.

\section{References}

Felix Berkenkamp and Markus Gwerder. Hybrid model predictive control of stratified thermal storages in buildings. Energy and Buildings, 84:233-240, 2014.

Eduardo F Camacho and Carlos Bordons Alba. Model predictive control. Springer Science \& Business Media, 2013.

Michaela Killian, Barbara Mayer, and Martin Kozek. Cooperative fuzzy model predictive control for heating and cooling of buildings energy and buildings. Energy and Buildings, 2016:130-140, 2015. URL doi:/10.1016/j.enbuild.2015.12.017.

Simeng Liu and Gregor P Henze. Impact of modeling accuracy on predictive optimal control of active and passive building thermal storage inventory. ASHRAE transactions, 110(1), 2004.

Lennart Ljung. System identification-theory for the user, 2nd edition ptr prentice hall. Upper Saddle River, NJ, 1999.

Yudong Ma, Francesco Borrelli, Brandon Hencey, Andrew Packard, and Scott Bortoff. Model predictive control of thermal energy storage in building cooling systems. In Decision and Control, 2009 held jointly with the 2009 28th Chinese Control Conference. CDC/CCC 2009. Proceedings of the 48th IEEE Conference on, pages 392-397. IEEE, 2009.

Barbara Mayer, Michaela Killian, and Martin Kozek. Management of hybrid energy supply systems in buildings 
using mixed-integer model predictive control. Energy Conversion and Management, 98:470-483, 2015. URL doi:/10.1016/j.enconman.2015.02.076.

Barbara Mayer, Michaela Killian, and Martin Kozek. A branch and bound approach for building cooling supply control with hybrid mpc. Energy and Buildings, 128:553-566, 2016. URL doi:/10.1016/j.enbuild.2016.07.027.

Frauke Oldewurtel, Alessandra Parisio, Colin N Jones, Dimitrios Gyalistras, Markus Gwerder, Vanessa Stauch, Beat Lehmann, and Manfred Morari. Use of model predictive control and weather forecasts for energy efficient building climate control. Energy and Buildings, 45:15-27, 2012.

Luis Pérez-Lombard, José Ortiz, and Christine Pout. A review on buildings energy consumption information. Energy and buildings, 40(3):394-398, 2008. URL doi:/10.1016/j.enbuild.2007.03.007.

Samuel Privara, Jiří Cigler, Zdeněk Váňa, Frauke Oldewurtel, Carina Sagerschnig, and Eva Žáčeková. Building modeling as a crucial part for building predictive control. Energy and Buildings, 56:8-22, 2013.

Jan Širokỳ, Frauke Oldewurtel, Jiří Cigler, and Samuel Prívara. Experimental analysis of model predictive control for an energy efficient building heating system. Applied Energy, 88 (9):3079-3087, 2011. 